\title{
Optimization of CPMG sequences to measure NMR transverse relaxation time $T_{2}$ in borehole applications
}

\author{
M. Ronczka and M. Müller-Petke \\ Leibniz Institute for Applied Geophysics, Hannover, Germany \\ Correspondence to: M. Ronczka (mathias.ronczka@liag-hannover.de) \\ Received: 3 May 2012 - Published in Geosci. Instrum. Method. Data Syst. Discuss.: 19 July 2012 \\ Revised: 9 October 2012 - Accepted: 25 October 2012 - Published: 21 November 2012
}

\begin{abstract}
Nuclear Magnetic Resonance (NMR) can provide key information such as porosity and permeability for hydrological characterization of geological material. In particular the NMR transverse relaxation time $T_{2}$ is used to estimate permeability since it reflects a pore-size dependent relaxation process. The measurement sequence (CPMG) usually consists of several thousands of electromagnetic pulses to densely record the relaxation process and to avoid relaxation processes that are due to diffusion. These pulses are equidistantly spaced by a time constant $t_{\mathrm{E}}$.
\end{abstract}

In NMR borehole applications the use of CPMG sequences for measuring the transverse relaxation time $T_{2}$ is limited due to requirements on energy consumption. For measuring $T_{2}$, it is state-of-the-art to conduct at least two sequences with different echo spacings $\left(t_{\mathrm{E}}\right)$ for recording fast and slow relaxing processes that correspond to different pore-sizes. We focus on conducting only a single CPMG sequence and reducing the amount of energy while obtaining both slow and fast decaying components and minimizing the influence of relaxation due to diffusion. Therefore, we tested the usage of CPMG sequences with an increasing $t_{\mathrm{E}}$ and a decreasing number of pulses.

A synthetic study as well as laboratory measurements on samples of glass beads and granulate material of different grain size spectra were conducted to evaluate the effects of an increasing $t_{\mathrm{E}}$. We show that $T_{2}$ distributions are broadened if the number of pulses is decreasing and the mean grain size is increasing, which is mostly an effect of a significantly shortened acquisition time. The shift of $T_{2}$ distributions to small decay times as a function of $t_{\mathrm{E}}$ and the mean grain size distribution is observed.

We found that it is possible to conduct CPMG sequences with an increased $t_{\mathrm{E}}$. According to the acquisition time and increasing influence of relaxation due to diffusion, the sequence parameters need to be chosen carefully to avoid misinterpretations.

\section{Introduction}

The method of nuclear magnetic resonance (NMR), discovered in the forties of the last century (Bloch et al., 1946; Purcell et al., 1946), has found widespread use in scientific and daily life. It was not later than the sixties that a first borehole tool was developed (Brown and Gamson, 1960) to take advantages of the unique properties of NMR in geophysics. It allows a direct detection and quantification of water since the initial amplitude of an NMR signal corresponds to the amount of protons exited. Furthermore, the measured relaxation times (longitudinal relaxation time $T_{1}$, transverse relaxation time $T_{2}$ ) depend on the pore geometry and can therefore be used to estimate hydraulic permeabilities (Seevers, 1966).

In laboratory-NMR, artificial magnetic fields and sequences to measure $T_{2}$ were early available. These CPMG sequences (Carr and Purcell, 1954; Meiboom and Gill, 1958) with several thousand pulses and a short equidistant pulse spacing $\left(t_{\mathrm{E}}\right)$ allow for densely recording the decaying signal, ensuring a sufficient acquisition time and minimize relaxation processes due to diffusion. Each of these pulses causes an NMR echo to be the measured signal (Hahn, 1950). Thus, $t_{\mathrm{E}}$ is referred to as the echo spacing of a CPMG sequence.

In borehole-NMR, there has been significant changes in the design of borehole tools in the nineties (Miller et al., 1990) using artificial magnetic fields and measuring $T_{2}$ using a CPMG. This made NMR a frequently used tool in oil and gas exploration. However, due to limitations on available 
pulse power for conduction of several thousands of pulses, until now it is common to obtain $T_{2}$ distribution based on conducting at least two different CPMG sequences in NMR borehole measurements. While slow decaying components are acquired with a sequence consisting of 1000 pulses and a large echo spacing $\left(t_{\mathrm{E}}\right)$, fast decaying components can be measured with only a few dozen echos and short echo spacing (Kruspe et al., 2009). Recently and in addition to obtaining $T_{2}$, there is an increasing interest in measuring multidimensional distributions involving diffusion and relaxation based on sequences, such as presented in Hürlimann and Venkataramanan (2002).

Beside applications in oil and gas exploration, NMR borehole measurements are needed in near-surface groundwater investigation. There are several reasons NMR logging has just started for this purpose. Standard NMR logging of groundwater monitoring wells is far too expensive with common oil and gas tools and service. Monitoring wells are of diameters as small as two inches, so a large diameter NMR tool cannot be used. A first commercially available small diameter NMR tool was introduced by Vista-Clara in 2011. This tool is designed for slimhole boreholes and hydrogeological investigations. An external surface station provides the necessary pulse power from the surface to the probe that is lowered into the well. This currently limits the tool to borehole depths of about $220 \mathrm{~m}$ (Vista Clara, 2011). The Larmor frequency for this tool can be switched between 245 and $290 \mathrm{kHz}$. This ensures a sufficient penetration depth $(190 \mathrm{~mm}$ from the tool center), even for drilling in unconsolidated material. Nevertheless, NMR measurements conducted with a low Larmor frequency provide a low signal-to-noise ratio, which has to be compensated by a high number of stacks. This leads to logging usually performed as point measurements. In cases of shallow boreholes and unconsolidated material that need sufficient penetration depth to obtain reliable results, this is probably the best way to design an NMR borehole tool; however, it is not applicable for deeper boreholes and incompatible with standard logging equipment.

It is this huge energy needed for CPMG pulse sequences that limits the construction of a small diameter tool that is interchangeable with logging equipment usually used in nearsurface logging business. Additionally, if a borehole tool should operate in depths up to $1000 \mathrm{~m}$, it is inevitable that all electronics for generating a NMR pulse sequence have to be implemented in the borehole tool. Therefore, research for developing an NMR borehole tool to characterize groundwater layers is still needed.

At the current state, we do not focus on a development of sequences that allow for estimating multi-dimensional relationships of diffusion and relaxation. To our knowledge a single CPMG measurement will not be sufficient for this purpose. We focus on conducting a single CPMG sequence that allows for obtaining slow and fast decaying components, minimizing the influence of relaxation due to diffusion and reducing the amount of energy. This can be reached by reducing the number of pulses and adjusting the echo spacing $\left(t_{\mathrm{E}}\right)$ between the pulses. We evaluated our optimized CPMG sequence with different parameter sets and various ways of increasing $t_{\mathrm{E}}$ in a synthetic study. The synthetic study is based on simple $T_{2}$ relaxation processes. We are aware of complex spin dynamic that may occur due to changed echo spacing within one sequence. But numerical modeling based on the modified Bloch-Torrey equation (Torrey, 1956) would be beyond the scope of this paper. However, to prove our findings we conducted laboratory experiments. In order to have sufficient control on pore-size, laboratory measurements were conducted on samples of glass beads and granular material of different grain size spectra. This helps to cover a broad range of material that may be observed in field experiments.

\section{$2 \mathrm{NMR}-\boldsymbol{T}_{2}$ relaxation}

\subsection{Free induction decay}

As a property of matter, namely the spin, a hydrogen proton posses a microscopic magnetic moment. In a static magnetic field $B_{0}$, a torque is applied that leads to an orientation of the magnetic moments along the stream lines of that field. According to the field strength of $B_{0}$, the protons precess with the Larmor frequency $\omega_{\mathrm{L}}=\gamma \cdot B_{0}$ about the stream lines of $B_{0}$, where $\gamma\left[\mathrm{Hz} \mathrm{T}^{-1}\right]$ is the gyromagnetic ratio. If an electromagnetic pulse $\left(B_{1}\right)$ with the frequency $\omega_{\mathrm{L}}$ perpendicular to $B_{0}$ is applied, the protons can be flipped about an angle dependent on the pulse duration and amplitude. Immediately after termination of the excitation pulse, all affected protons return (relax) to the equilibrium state, i.e., the direction of the external static magnetic field $\left(B_{0}\right)$. During relaxation the protons precess about the stream lines of $B_{0}$. If the stream lines of $B_{0}$ denote the $\mathrm{z}$-direction, the relaxation process is observed in the $x-y$ plane. The decay in the $x-y$ plane can be described by Eq. (1).

$M_{\mathrm{xy}}(t)=M_{0} \cdot e^{\left(\frac{-t}{T_{2}}\right)}$

Here, $M_{0}$ is the initial amplitude, $T_{2}$ the transverse relaxation time and $M_{\mathrm{xy}}$ the magnetisation in the x-y plane. For further information on basic NMR theory we refer to Dunn et al. (2002) or Coates et al. (1999). Basic parameters of interest are the initial amplitude $M_{0}$ at $t=0$, due to its sensitivity to water content and the relaxation time $T_{2}$ as an indicator of pore space properties. Hence, the transverse relaxation time $T_{2}$ denotes how fast or slow the magnetisation decreases in the $x-y$ plane.

However, the relaxation process in the $x-y$ plane might be significantly influenced by inhomogeneities in the magnetic field, i.e., magnetic gradients. These inhomogeneities can be on macro scale like variations in the field strength of $B_{0}$, or small scaled due to particles with a significant magnetic 
susceptibility in the porous medium. Variations of the magnetic field intensities lead to slightly different Larmor frequencies and therefore to a loss of phase coherence in the $x-y$ plane. The precession of protons in the $\mathrm{x}-\mathrm{y}$-plane under the influence of magnetic gradients is illustrated schematically in Fig. 1. At $t=t_{0}$ all protons are coherent. The slightly different Larmor frequencies lead to a dephasing of the protons, which results in a reduced transverse magnetization. This relaxation time observed is mostly referred to as $T_{2}^{*}$ (Dunn et al., 2002).

This relaxation time $T_{2}^{*}$ of water in porous media consists of three parts: the bulk relaxation $T_{2, \mathrm{~B}}$, the surface relaxation $T_{2, \mathrm{~S}}$, and the relaxation due to any kind of dephasing $T_{2, \mathrm{P}}$. All parts are parallel processes and sum up to $T_{2}^{*}$, the relaxation time of the free induction decay (FID) as decribed in Eq. (2) (Mohnke and Yaramanci, 2008).

$$
\frac{1}{T_{2}^{*}}=\frac{1}{T_{2, \mathrm{~B}}}+\frac{1}{T_{2, \mathrm{~S}}}+\frac{1}{T_{2, \mathrm{P}}}
$$

The bulk relaxation $T_{2, \mathrm{~B}}$ denotes the relaxation time of free water, the surface relaxation $T_{2, \mathrm{~S}}$ describes any influences of the pore surface to the protons in the vicinity of the surface, and $T_{2, \mathrm{P}}$ includes all kinds of magnetic gradients (macroscopic, microscopic).

\section{$2.2 \quad T_{2}$-relaxation}

By conducting a CPMG sequence (Carr and Purcell, 1954; Meiboom and Gill, 1958), influences of magnetic gradients can be minimized. Due to the CPMG sequence, $T_{2, \mathrm{P}}$ can be simplified to a diffusion term $T_{2, \mathrm{D}}$. The relaxation due to diffusion $T_{2, \mathrm{D}}$ occurs if a proton moves through magnetic gradients either macroscopic or microscopic. While moving, the proton passes through regions with varying magnetic field strengths, which results in changing Larmor frequencies. According to different pathways of single protons within a set of protons and therefore differences in the Larmor frequencies, the set of protons lose their phase coherence. This diffusion effect is non-removable. The diffusion term can be expressed via Eq. (3) (e.g., Kleinberg and Horsfield, 1990):

$$
\frac{1}{T_{2, \mathrm{D}}}=D \frac{\left(\gamma G t_{\mathrm{E}} / 2\right)^{2}}{3},
$$

with the gradient $G\left[\mathrm{G} \mathrm{cm}^{-1}\right]$ of the magnetic field, $\gamma$ $\left[\mathrm{Hz} \mathrm{T}^{-1}\right]$ the gyromagnetic ratio, $D\left[\mathrm{~m}^{2} \mathrm{~s}^{-1}\right]$ the diffusion coefficient and $t_{\mathrm{E}}[\mathrm{s}]$ the spacing between an echo and the inducing pulse. As $T_{2, \mathrm{P}}$ can be reduced to $T_{2, \mathrm{D}}$, Eq. (2) transforms to Eq. (4), which now describes all effects that influences the $T_{2}$ decay time.

$$
\frac{1}{T_{2}}=\frac{1}{T_{2, \mathrm{~B}}}+\frac{1}{T_{2, \mathrm{~S}}}+D \frac{\left(\gamma G t_{\mathrm{E}} / 2\right)^{2}}{3}
$$

According to Eq. (3), diffusion effects can be minimized by choosing a small $t_{\mathrm{E}}$ while conducting a CPMG sequence.

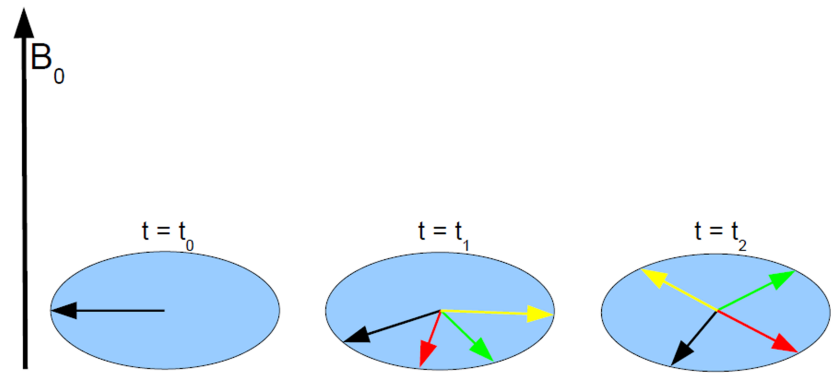

Fig. 1. Loss of phase coherence of protons due to differences in the Larmor frequency in the presence of magnetic gradients.

Equation (3) also indicates that diffusion effects take more effect if the echo spacing $t_{\mathrm{E}}$ is too large. If the diffusion term can be assumed to be negligible, only $T_{2, \mathrm{~B}}$ and $T_{2, \mathrm{~S}}$ affect the decay time in Eq. (4).

\subsection{Estimation of pore size by means of $T_{2}$}

According to Eq. (4), the transverse relaxation time $T_{2}$ measured by a CPMG sequence consists of $T_{2, \mathrm{~B}}$ and $T_{2, \mathrm{~S}}$. The bulk relaxation $T_{2, \mathrm{~B}}$ denotes the relaxation of free water. Here the protons are only affected by other protons during relaxation. The surface relaxation $T_{2, \mathrm{~S}}$ includes all effects of the grain surface that lead to a faster relaxation. These effects imply magnetic properties and the structure of the inner surface of the material. The surface relaxation, assuming a fast diffusion regime (Brownstein and Tarr, 1979), can be expressed by Eq. (5):

$$
\frac{1}{T_{2, \mathrm{~S}}}=\rho \frac{S}{V}
$$

whereas $\rho\left[\mathrm{m} \mathrm{s}^{-1}\right]$ denotes the surface relaxivity, $S\left[\mathrm{~m}^{2}\right]$ the surface area and $V\left[\mathrm{~m}^{3}\right]$ the volume of a pore. In a porous media, $\mathrm{V}$ and $\mathrm{S}$ refer to the volume and the surface area of the whole sample. In this case the surface relaxivity is a mean value of all water-filled pores. According to Eq. (5), the influence of surface relaxation increases for small pores and/or large surface relaxivities. Due to these dependencies fine material with small pores will create a faster decaying signal compared to coarse material. Thus, by knowing the surface relaxivity $(\rho)$ of a sample it is possible to calculate a mean pore radius. If, for example, a spherical pore geometry is assumed, the surface to volume ratio $\left(\frac{S}{V}\right)$ equals $\frac{6}{d}$; or if a cylindrical geometry is assumed, $\frac{S}{V}=\frac{1}{d}$ (without top and bottom).

\section{CPMG sequences}

\subsection{Classic CPMG}

The CPMG sequence consists of an excitation pulse (P90), which flips the orientation of the macroscopic magnetisation 
about $90^{\circ}$ from the $\mathrm{z}$-direction into the $\mathrm{x}-\mathrm{y}$ plane and several refocusing pulses (P180), which flip the protons about $180^{\circ}$ in the $\mathrm{x}-\mathrm{y}$ plane. After the excitation pulse (P90), the protons lose their phase coherence due to slightly different Larmor frequencies caused by magnetic gradients. After a time span $\tau$, a refocusing pulse (P180) is applied, which flips the protons in a manner that those with a higher $\omega_{\mathrm{L}}$ lag behind those with a lower one. Due to refocusing, an echo is build up and reaches its maximum after $t_{\mathrm{E}}=2 \tau$. The maximum of the echo marks the point of maximal coherence of the protons.

In general, $\tau$ is constant over the whole CPMG sequence and has to be very small to ensure that the diffusion term is negligible, otherwise the protons are not refocused completely, which leads to a reduced amplitude of the echo. In laboratory measurements, $\tau$ is typically set between 100 $300 \mu$ s. In order to acquire the whole decay process a large number of echos $\left(n_{\text {echo }}\right)$ is needed. Commonly in laboratory measurements several thousand echos are performed within one CPMG sequence. The maximum of all echos detected gives the relaxation curve for estimating the $T_{2}$ relaxation time. A classical CPMG sequence is shown in Fig. 2a. Note that, if for a small $\tau$ diffusion can be neglected, then one can conduct a number of CPMG with different $\tau$ to measure diffusion processes.

\subsection{CPMG with variable $\tau$ spacing}

For the purpose of using NMR as a borehole tool, a significant reduction of energy is needed for conducting a CPMG due to logging requirements for transport and storage of energy. This is equivalent to a reduction of pulses used (accordingly $n_{\text {echo }}$ ) for a sequence. As a result of simply reducing the number of echos, the acquisition time is significantly shortened. This leads to errors in the estimated relaxation time if the decay process is not recorded completely. For compensation, i.e., to extent the acquisition time, the echo spacing $t_{\mathrm{E}}=2 \tau$ can be increased. As mentioned before, this would increase the diffusion effects according to Eq. (3) from the beginning of the sequence, which is not intended. Additionally, the density of data points that forms the $T_{2}$ decay curve is reduced. Thus, it can happen that too few data points in the beginning of the decay curve are not sufficient for detecting fast decaying components. However, a CPMG with an increased constant $\tau$ causes insufficient sampling of fast decaying components in the beginning of the decay curve. Thus, it can be expected that a wrong decay time could be estimated. Additionally, diffusion effects will increase too, according to Eq. (3).

We investigated the effect of different variable $\tau$ spacings on the estimated decay time. We designed CPMG sequences with a variable $\tau$, as schematically depicted in Fig. $2 \mathrm{~b}$. The important parameters for constructing a CPMG sequences with a variable $\tau$ spacing are the number of echos $\left(n_{\text {echo }}\right)$ and a minimum and maximum $\tau$. For generating a sequence with an increasing $\tau$ we fixed the values of $\tau_{\min }$ and $\tau_{\max }$.
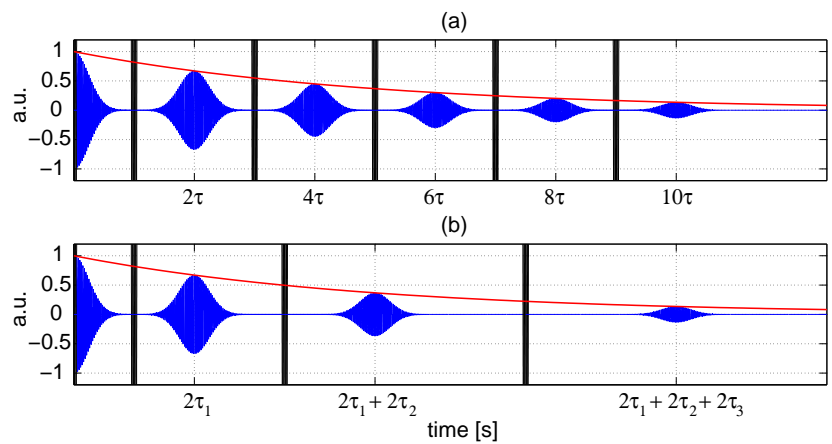

Fig. 2. (a) Classical CPMG sequence with a P90 excitation pulse and several P180 pulses for refocusing. After each P180 pulse an echos appears. The envelope of all detected echos gives the $T_{2}$ decay curve. (b) First pulses and the resulting echos for a CPMG sequence with variable $\tau$ spacing.

Between these, an amount of $n_{\text {echo }}$ linearly increased; exponentially equidistant and logarithmically equidistant $\tau$ is calculated. Starting with the lowest possible $\tau_{\min }\left(\tau_{1}\right.$ in Fig. $2 b$ ), the fast decaying components are sampled as accurately as possible, while the components with a slow decay are already appropriately sampled.

For the sake of clarity, the development of single CPMG sequence with a variable echo spacing does not allow for estimating diffusion or internal gradient at pore scale like experiments as described in Hürlimann and Venkataramanan (2002) or Song (2003). The intension of this optimized CPMG is to measure $T_{2}$ as appropriate as possible under the scope of reducing the number of pulses.

\section{Synthetic study}

We conducted a synthetic study to test the evolution of errors for the estimated transverse relaxation time $T_{2}$ from CPMG sequences with different approaches of increasing $\tau$. First we generated synthetic data of a simple mono-exponential signal to get an overview of the different sequences. Secondly a synthetic multi-exponential signal was generated to evaluate if fast and slow decaying parts are distinguishable and can be determined correctly. Therefore, we used a bi-exponential signal with the transverse relaxation times $T_{2,1}$ and $T_{2,2}$ and fitted the data via a decay time distribution. Finally, to examine influences of diffusion on the data due to an increased $\tau$, a diffusive part was added to the synthetic data.

We should note that forward modeling based on Eq. (4) is not the most rigorous approach to evaluate the influence of increasing $\tau$. Changing the symmetry, i.e., the constant echo spacing, may cause complex spin dynamics that can be studied based on the Bloch-Torrey equation (Torrey, 1956). However, this would be beyond the scope of this paper. We decided to support our finding of the synthetic study by conducting laboratory-NMR experiments. 

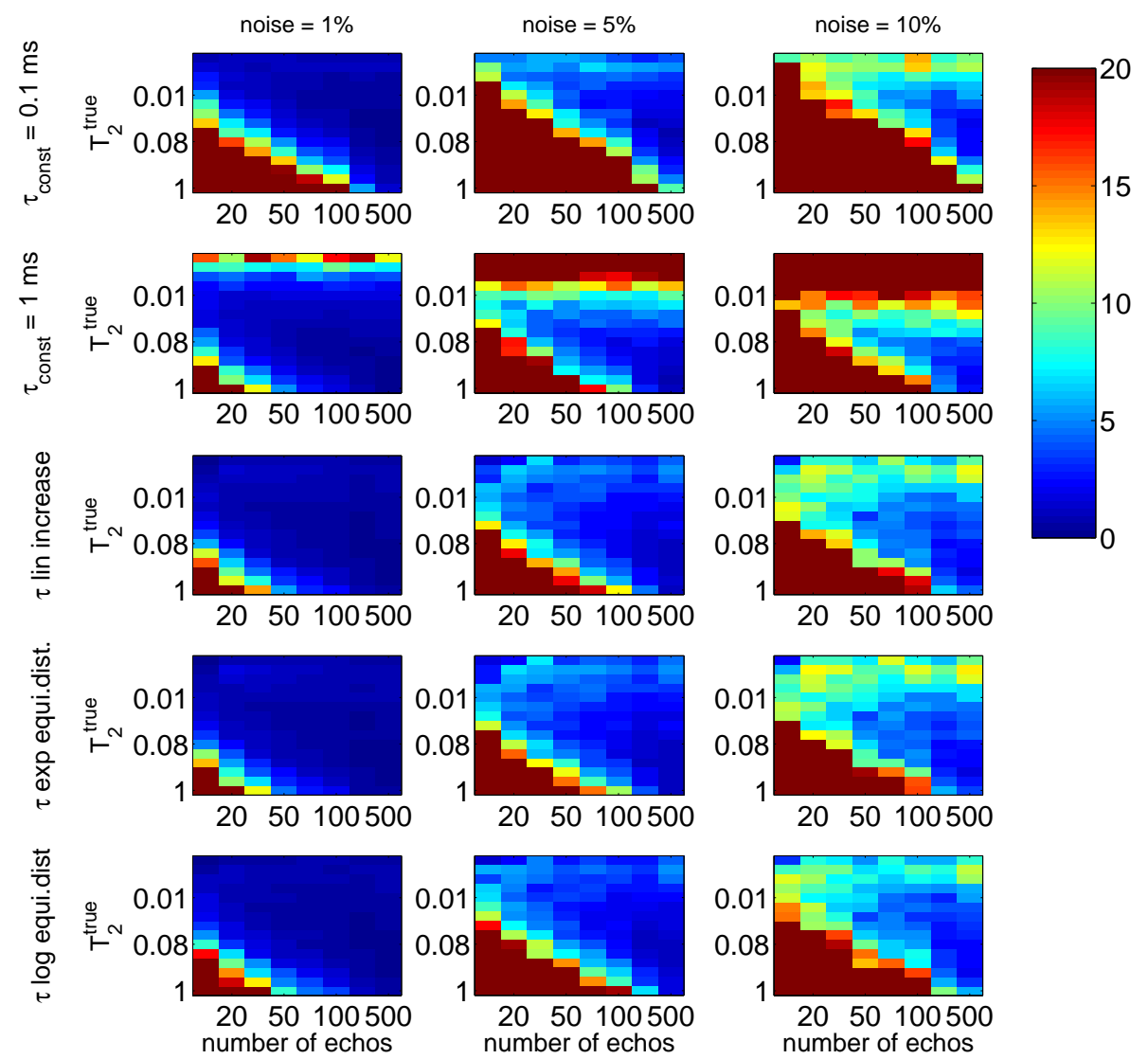

Fig. 3. The evolution of error for different signal-to-noise ratio. Standard deviations between the true transverse decay time $T_{2}^{\text {true }}$ and the estimated $T_{2}$ depending on the number of echos for mono-exponential synthetic data is shown. All errors over $20 \%$ are red coloured.

\subsection{Mono-exponential signal}

A mono-exponential signal can be generated using Eq. (1). The set of model parameters are the initial transverse magnetisation $M_{0}$ and the transverse decay time $T_{2}$. Here, $M_{0}$ is set to 1 and the decay time for generating the synthetic data is named $T_{2}^{\text {true }}$ and is varied between $1 \mathrm{~ms}$ and $1 \mathrm{~s}$. Standard deviations between the true transverse decay time $T_{2}^{\text {true }}$ (fixed model parameter) and the estimated $T_{2}$ of CPMG sequences with a variable $\tau$ spacing were calculated for three different noise levels and are plotted in Fig. 3, each as a function of the true decay time $\left(T_{2}^{\text {true }}\right)$ and $n_{\text {echo. }}$. All deviations above $20 \%$ are red coloured.

The results for sequences conducted with a small and large constant $\tau$ are displayed in the first two rows of Fig. 3. As expected, the error of the estimated decay times increases if $n_{\text {echo }}$ decreases or the true decay time increases, i.e., slow decaying signals. This is a result of an insufficient acquisition time. If the noise increases, the range of high errors is extended to higher $n_{\text {echo }}$ and smaller $T_{2}^{\text {true }}$ (see first row in Fig. 3). Additional errors for small decay times occur if the echo spacing $(\tau)$ is increased (second row of Fig. 3), which is probably caused by a bad sampling of fast decaying signals.
Thus, increasing a constant $\tau$ to compensate for the reduction of $n_{\text {echo }}$ is not an appropriate approach.

All three sequences with a variable $\tau$ spacing, illustrated in the last three rows of Fig. 3, exhibit more or less the same behaviour for the range of errors. Comparing CPMG-sequences with a variable $\tau$ spacing with the one using a constant small $\tau$ (Fig. 3, first row), smaller errors occur for decreasing $n_{\text {echo }}$ due to the elongated acquisition time. Compared to the sequence with a constant large $\tau$ (Fig. 3, second row), all optimized sequences show smaller errors for fast decaying signals, which is a result of a tighter sampling of the decay curve at early times. As the noise level increases, the errors over $20 \%$ expand to sequences with larger $n_{\text {echo }}$ and smaller decay times too.

\subsection{Bi-exponential signal}

In the next step a bi-exponential signal was generated to examine if both the fast and the slow decaying parts of the signal can be estimated while reducing $n_{\text {echo }}$. For generating the synthetic data, Eq. (1) was extended to a sum of two exponentials with the transverse decay times $T_{2,1}$ and $T_{2,2}$. The decay times were chosen in a manner that they are far apart from each other. Therefore, the synthetic decay curve 

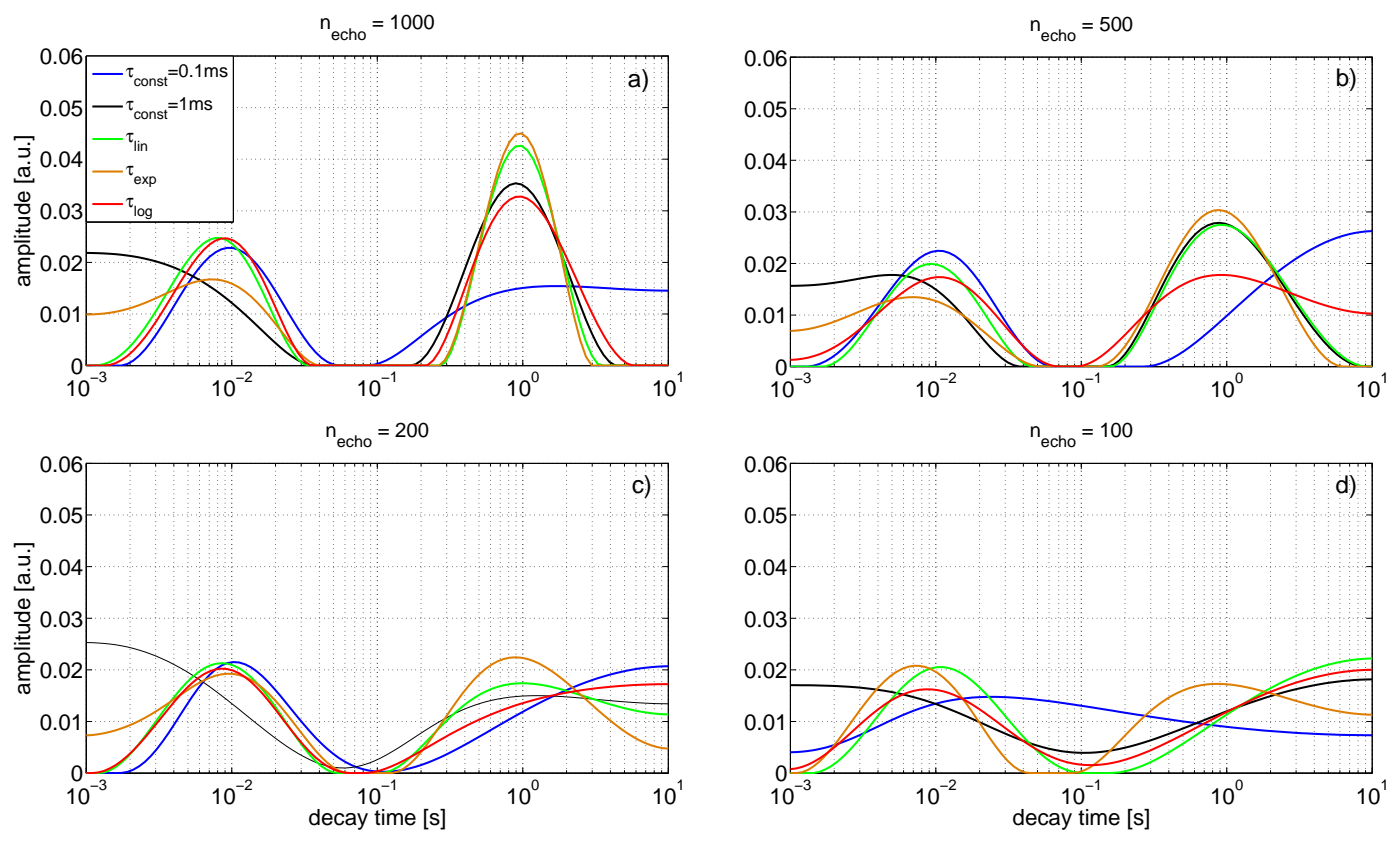

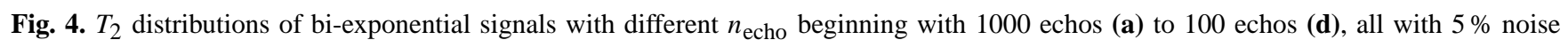
added. Sequences with linearly increasing, exponentially equidistant and logarithmically equidistant $\tau$ spacing with $\tau$ max $=2 \mathrm{~ms}$ are plotted. Additionally two sequences with an constant $\tau$ spacing are shown.

is composed of the decay times $T_{2,1}=0.01 \mathrm{~s}$ and $T_{2,2}=1 \mathrm{~s}$ with $5 \%$ noise added. For all sequences with different variable $\tau$ spacing, the recorded $n_{\text {echo }}$ were reduced successively from 1000 to 100 echos.

The decay curves were fitted with a multi-exponential approach using an inverse Laplace transformation. This calculates corresponding amplitudes to a predefined amount of decay times. For smoothness constraints a Tikhonov regularisation of first kind was used. The regularisation parameter was determined via the L-curve criterion that gives a solution closest to the corner of the L-curve (Aster et al., 2005). The $T_{2}$ distributions of the fitted signals are illustrated in Fig. 4 and consist of 100 logarithmically-equidistant-spaced decay times within a range of $T_{2} \in\left[10^{-3}, 10^{1}\right]$.

In Fig. 4a the signal is sampled with $n_{\text {echo }}=1000$. In all curves a bi-exponential behaviour is observable. Sequences with a small constant $\tau$ (blue curve in Fig. 4) cannot resolve the slow decaying parts of the signal due to a short acquisition time (about $0.2 \mathrm{~s}$ ). As a result the $T_{2}$ distribution is broadened and decay times above $3 \mathrm{~s}$ are fitted, which exceeds the maximal possible transverse decay time $\left(T_{2}\right)$ of water. Thus, a logarithmic mean decay time of this $T_{2}$ distribution will be overestimated.

In contrast, fast decaying components of the signal cannot be resolved by a CPMG sequence with a large constant $\tau$ spacing (see the black curve in Fig. 4a). This can also be seen in Kleinberg (1994).

Both parts of the signal can be more or less resolved by all sequences with a variable $\tau$ spacing. The sequence with an exponentially equidistant $\tau$ spacing shows a broadened $T_{2}$ distribution for fast decaying components, which is a result of a fast increasing $\tau$ that lead to an insufficient sampling of the decay curve for early times.

In general, if $n_{\text {echo }}$ decreases, the bi-exponential behaviour vanishes in conjunction with a broadened $T_{2}$ distribution due to the shortened acquisition time. If $n_{\text {echo }}$ is reduced to 200 echos (see Fig. 4c), only sequences with a linearly increased and exponentially-equidistant $\tau$ spacing exhibit a maximum for slow decaying components. The fast decaying component is resolvable by all sequences.

\subsection{Bi-exponential signal with diffusion}

According to Eq. (4), the $\tau$ spacing is the only sequence parameter that influences the diffusion term of an NMR signal. Thus, different kinds of increasing $\tau$ lead to different impacts of the diffusion on the measured signal. To examine this effect we added a diffusion component to the signal.

According to Coates et al. (1999), the magnetic gradient is influenced by three factors. One depends on the tool design (i.e., tool size, frequency and shape of the used magnet). The second one handles conditions of the formation, such as the temperature. The third factor is described by gradients induced by the applied magnetic field, which are the result of differences in the magnetic susceptibility of grains and pore fluid and are referred to as internal gradients. The magnetic gradient of an MRIL tool (Coates et al., 1999) is denoted with $17 \mathrm{G} \mathrm{cm}^{-1}$, but Kenyon (1997) stated that a common magnetic gradient from commercial NMR logging tools are 

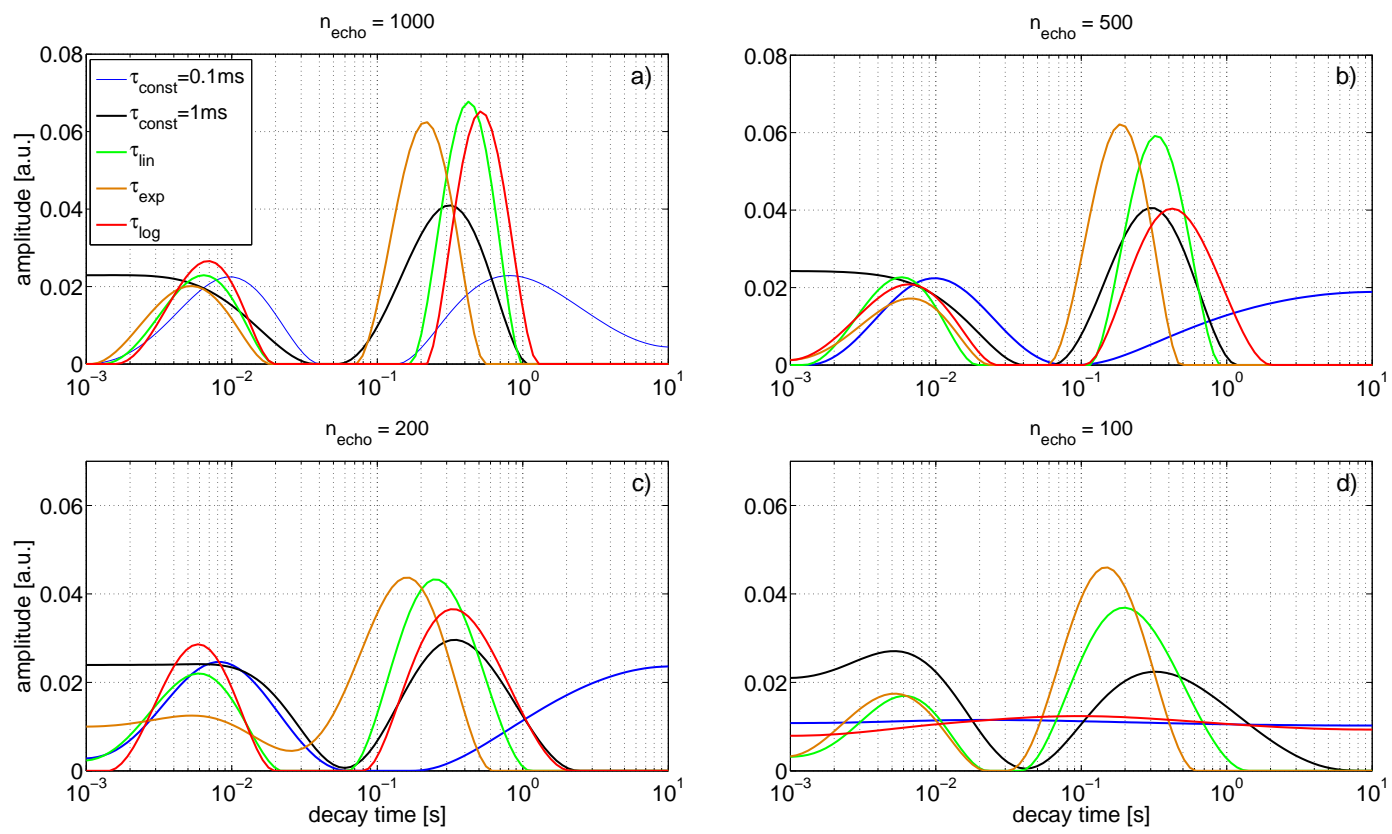

Fig. 5. $T_{2}$ distribution of a bi-exponential signal with $T_{2,1}=0.01 \mathrm{~s}$ and $T_{2,2}=1 \mathrm{~s}$ with a diffusion component added and $5 \%$ noise. CPMGs with linearly increasing, exponentially equidistant and logarithmically equidistant $\tau$ spacing with $\tau_{\max }=2 \mathrm{~ms}$ are plotted for different amounts of echos. Additionally, two sequences with an constant $\tau$ spacing are plotted.

about $20 \mathrm{G} \mathrm{cm}^{-1}$. Thereby, we set $G$ to $18 \mathrm{G} \mathrm{cm}^{-1}$ as a realistic magnetic gradient of the $B_{0}$ field. Furthermore, the diffusion constant of water $D=2.5 \times 10^{-9} \mathrm{~m}^{2} \mathrm{~s}^{-1}$ was used. The different impacts of $G$ are included by the increased $\tau$. The $T_{2}$ distributions for the CPMG sequences with different variable $\tau$ spacings and different $n_{\text {echo }}$ are plotted in Fig. 5.

Compared to the non-diffusive signal (Fig. 4), all $T_{2}$ distributions in Fig. 5 are shifted to smaller decay times, except the sequence with constant $\tau=0.1 \mathrm{~ms}$ (blue curve in Fig. 5). As mentioned before, there are only small influences of diffusion relaxation on the $T_{2}$ relaxation time for sequences with a short $\tau$ spacing. Nevertheless, the short acquisition time leads to a broadened decay spectrum, which leads to an overestimated log mean decay time. This is more pronounced if $n_{\text {echo }}$ is reduced (see Fig. 5b-d).

Thus, if a variable $\tau$ spacing is applied, $T_{2, \mathrm{D}}$ increases for increasing $\tau_{\max }$ and/or a fast increasing $\tau$. In addition it can be seen that slow decaying components are more influenced by diffusion than the faster ones. This is already visible in Eq. (4). Relatively small $\tau$ spacings in the beginning of the sequences lead to small influences of diffusion on the signal, but increases successively to larger $\tau$ spacing in the end of the sequence.

The largest shifts to short decay times are observable at $\mathrm{CPMG}_{\tau \text {,exp }}$ (see orange curve in Fig. 5), which has the fastest increasing $\tau$ spacing. The sequence with a logarithmically equidistant $\tau$ spacing $\left(\mathrm{CPMG}_{\tau}\right.$, log , red curve in Fig. 5) has the slowest increasing $\tau$ and therefore the smallest shift to short decay times. However, it also exhibits the shortest acquisition time, and thus leads to the most broadened distribution within the CPMG sequences with a variable $\tau$ spacing.

Considering the trade-off between effects due to short acquisition times (broadening) and diffusion (shift to smaller decay times), the CPMG sequence with a linearly increasing $\tau$ (Fig. 5, green curve) is superior compared to sequences with an exponentially and logarithmically equidistant $\tau$. Therefore, the CPMG sequence with a linearly increased $\tau$ was used for laboratory measurements.

\section{Laboratory measurements}

As it can be seen in Fig. 5, two main effects influence the estimated decay time distributions and thus the $T_{2}$ decay time: (i) a shortened acquisition time due to a reduction of $n_{\text {echo }}$, and (ii) an increased diffusion term caused by an increased inter-echo spacing $t_{\mathrm{E}}=2 \tau$. On the one hand, these have to be minimized for the widest possible grain size spectrum. On the other hand, the energy consumption for conducting a CPMG sequence has to be minimized in order to not exceed the possibilities of the borehole tool.

The requirement on estimating the $T_{2}$ decay time is a minimal or acceptable deviation to a reference sequence. Thus, optimal settings for $n_{\text {echo }}$ and $\tau_{\max }$ have to be found. For this purpose laboratory measurements with the Maran Ultra 8 (Resonance Instruments) were conducted. Unfortunately, our instrument does not allow for constant gradients of the magnetic field to simulate gradients as expected from borehole 
Table 1. Grain size spectra of different samples for glass beads and granular material used in laboratory measurements, with $\mathrm{gU}$ - coarse silt; $\mathrm{mS}, \mathrm{gS}$ - medium, coarse sand.

\begin{tabular}{lll}
\hline & $\begin{array}{c}\text { glass beads } \\
\mathrm{d}[\mu \mathrm{m}]\end{array}$ & $\begin{array}{c}\text { granular material } \\
\mathrm{d}[\mu \mathrm{m}]\end{array}$ \\
\hline $\mathrm{gU}$ & $40-70$ & - \\
$\mathrm{mS}$ & $250-500$ & $250-500$ \\
$\mathrm{gS}$ & $1250-1650$ & $1000-1600$ \\
\hline
\end{tabular}

tools. Thus, the influence of diffusion that is observed belongs to internal gradients at pore scale and inhomogeneities of the artificial magnetic field.

\subsection{Sample preparation and parameter sets of CPMG sequences}

Samples of glass beads and granular material with grain size spectra varying from coarse silt $(\mathrm{gU})$ to coarse sand $(\mathrm{gS})$ were used. The grain size spectra of the used material are given in Table 1.

For each grain size distribution, three samples were prepared. The material was filled in small cylindrical vessels with a height of $h=2.6 \mathrm{~cm}$ and a diameter of $d=3.6 \mathrm{~cm}$. The sample material was trickled into the vessel, which was filled with distilled water. To assure comparability of porosity between the three samples of the same material, an ultrasonic bath was used to raise the packing density to a maximum. Afterwards the samples were filled up and sealed with a foil to minimize evaporation effects during NMR measurements. To avoid further heating during NMR measurements and thus temperature effects on the initial amplitude and the decay time (Godefroy et al., 2001), the samples were heated up to $25-30^{\circ} \mathrm{C}$. The samples were weighed before and after NMR measurements to determine if evaporation occurred during the NMR measurements. Variations of the mass less than $1 \%$ were observed.

If a sample is completely water saturated, the porosity can be calculated using the initial amplitude. A comparison between the NMR- and gravimetric-porosity is illustrated in Fig. 6, which is an easy way to determine if any faults were made during the preparation or measurement.

We performed 9 CPMG sequences per sample with different values of $\tau_{\max }$ and $n_{\text {echo. The logarithmic mean of }}$ the $T_{2}$ distribution was compared with the log mean decay time $\left(T_{2, \mathrm{lg}}\right)$ of the reference sequence, which consists of 5000 pulses and a constant $\tau$ of $300 \mu$ s. The pulse-echo spacing increased in a linear manner, staring with $\tau_{\min }=100 \mu \mathrm{s}$, while $\tau_{\max }$ was set between 400 and $4000 \mu$ s. The minimal echo spacing $\left(\tau_{\min }\right)$ was the lowest possible value that could be realized with the Maran Ultra 8 . The number of echos was decreased, beginning with $n_{\text {echo }}=500$ down to $n_{\text {echo }}=50$. By choosing these sequence parameters, an outline of the effect on the acquisition time together with diffusion effects for
Table 2. Sequence parameter for the tested CPMG sequences. Starting from a fixed $\tau_{\min }=100 \mu \mathrm{s}$, the echo spacing was increased up to $\tau_{\max }$, which equals to the total acquisition time $t_{\text {tot }}$ given in column 3 .

\begin{tabular}{lrl}
\hline$n_{\text {echo }}$ & $\tau_{\max }[\mathrm{ms}]$ & $t_{\text {tot }}[\mathrm{s}]$ \\
\hline \multirow{3}{*}{500} & 4000 & 2.05 \\
& 1000 & 0.55 \\
& 400 & 0.25 \\
\hline \multirow{3}{*}{200} & 4000 & 0.82 \\
& 1000 & 0.22 \\
& 400 & 0.1 \\
\hline \multirow{3}{*}{50} & 4000 & 0.205 \\
& 1000 & 0.055 \\
& 400 & 0.025 \\
\hline
\end{tabular}

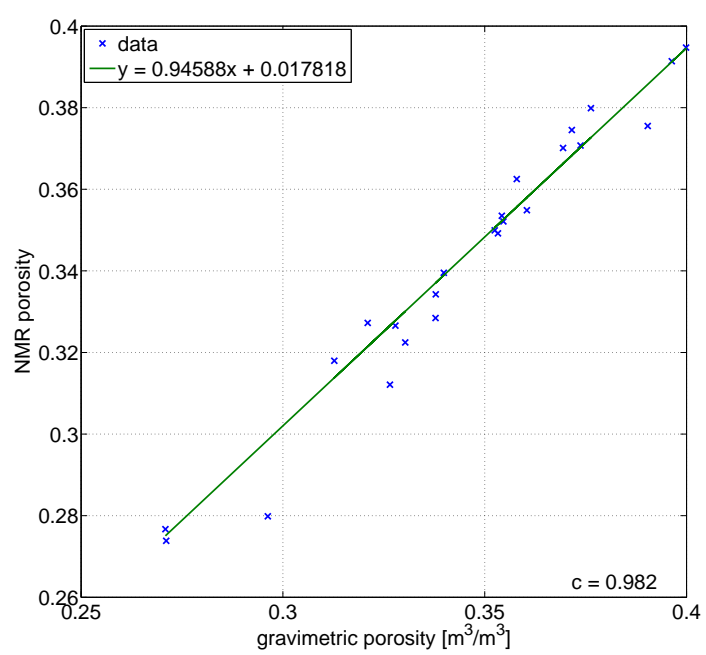

Fig. 6. Cross-plot of porosities determined via NMR- and gravimetric-measurements. The NMR-porosity is in excellent agreement with the gravimetric ones, which is validated by the correlation coefficient of $c=0.982$.

different grain sizes, i.e., signals with different decay times, could be obtained. A full list of the main sequence parameters $n_{\text {echo }}$ and $\tau_{\max }$ together with the resulting acquisition time $t_{\text {tot }}$ is given in Table 2 .

\subsection{Results}

\subsection{1 $\quad T_{2}$ distribution of glass beads}

The $T_{2}$ distribution of different glass beads are illustrated in Fig. 7. The mean grain size spectra increases from top to bottom, in which samples of coarse silt $(40-70 \mu \mathrm{m})$ are depicted in the first row (Fig. 7a-c), samples of medium sand $(250-500 \mu \mathrm{m})$ in the second row (Fig. 7d-f) and samples of coarse sand $(1250-1650 \mu \mathrm{m})$ in the third row. The echos decrease from left to right, starting with $n_{\text {echo }}=500$ down to 
Table 3. Logarithmic mean decay time $T_{2, \mathrm{lg}}$ for all CPMG sequences conducted on glass beads. The second column denotes $T_{2, \mathrm{lg}}$ of the reference sequence conducted with $n_{\text {echo }}=5000$ and a constant $\tau=300 \mu \mathrm{s}$.

\begin{tabular}{lcrrrr}
\hline \multicolumn{1}{c}{ sample } & $\begin{array}{c}T_{2, \lg }[\mathrm{ms}] \\
(\text { reference })\end{array}$ & $\tau_{\max }[\mu \mathrm{s}]$ & $\begin{array}{c}n_{\text {echo }}: 500 \\
\left(T_{2, \text { lg }}[\mathrm{ms}]\right)\end{array}$ & $\begin{array}{c}200 \\
\left(T_{2, \text { lg }}[\mathrm{ms}]\right)\end{array}$ & $\begin{array}{c}50 \\
\left(T_{2, \text { lg }}[\mathrm{ms}]\right)\end{array}$ \\
\hline silt & & 400 & 91.55 & 81.78 & 75.02 \\
$(40-70 \mu \mathrm{m})$ & 73.25 & 1000 & 76.91 & 68.22 & 53.40 \\
& & 4000 & 59.38 & 46.7 & 30.85 \\
\hline medium-grained & & 400 & 592.58 & 761.54 & 1083.6 \\
$(250-500 \mu \mathrm{m})$ & 487.68 & 1000 & 508.07 & 461.41 & 454.01 \\
& & 4000 & 457.52 & 424.97 & 378.58 \\
\hline coarse-grained & & 400 & 1721.7 & 1869.1 & 1824.6 \\
$(1250-1650 \mu \mathrm{m})$ & 1320.1 & 1000 & 1543.1 & 1728.4 & 2057.6 \\
& & 4000 & 1366.6 & 1589.1 & 1405.8 \\
\hline
\end{tabular}

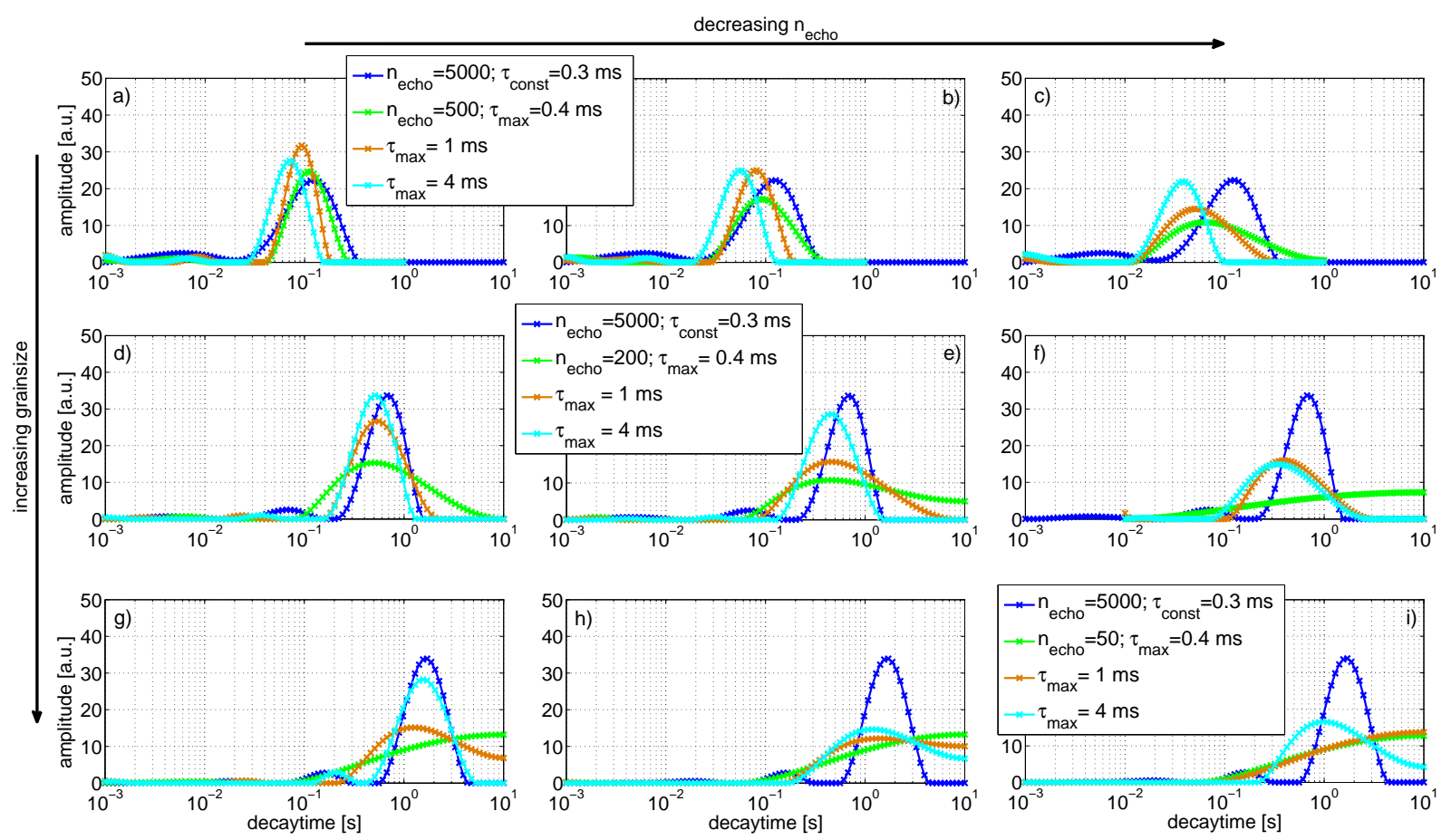

Fig. 7. $T_{2}$ distribution of glass beads for CPMG sequences with a linearly increased $\tau$. Columns indicate a decreasing $n_{\text {echo }}(500-200-50$, also given in the legends). The grain size increases from top to bottom (first row - coarse silt; second row - fine sand; third row - coarse sand).

$n_{\text {echo }}=50$. Within each subplot of Fig. 7, the blue coloured $T_{2}$ distribution is associated with the reference sequence. The other curves denote CPMG sequences with a linearly increasing $\tau$ spacing and different $\tau_{\max }$ values. The corresponding $\log$ mean decay times of the distribution are given in Table 3 .

For $n_{\text {echo }}=500$ (Fig. 7a) the $T_{2}$ distribution for finegrained glass beads are displayed. Compared to the reference sequence a shift of the main peaks to smaller decay times is observed, which is more distinctive for an increasing $\tau_{\max }$. For a constant $n_{\text {echo }}$ the $\tau$ spacing increases faster for larger $\tau_{\max }$. Thus, considering Eq. (3), a larger $\tau$ leads to a higher attenuation due to diffusion and results in a larger shift to smaller decay times.

For $n_{\text {echo }}=500$ and increasing grain size (from top to bottom), it shows that a shift to small decay times is observed for all grain sizes. For the fine-grained sample (Fig. 7a) the difference of the main peak to the reference signal is about $55.65 \mathrm{~ms}$ considering the sequence with $\tau_{\max }=4000 \mathrm{~ms}$, while the shift for the medium-grained sample (Fig. 7d) is (with a difference of $164 \mathrm{~ms}$ ) increased. In contrast, for coarse sand samples the shift decreases with a larger $\tau_{\max }$, which is due to the short acquisition time. For coarse-grained 

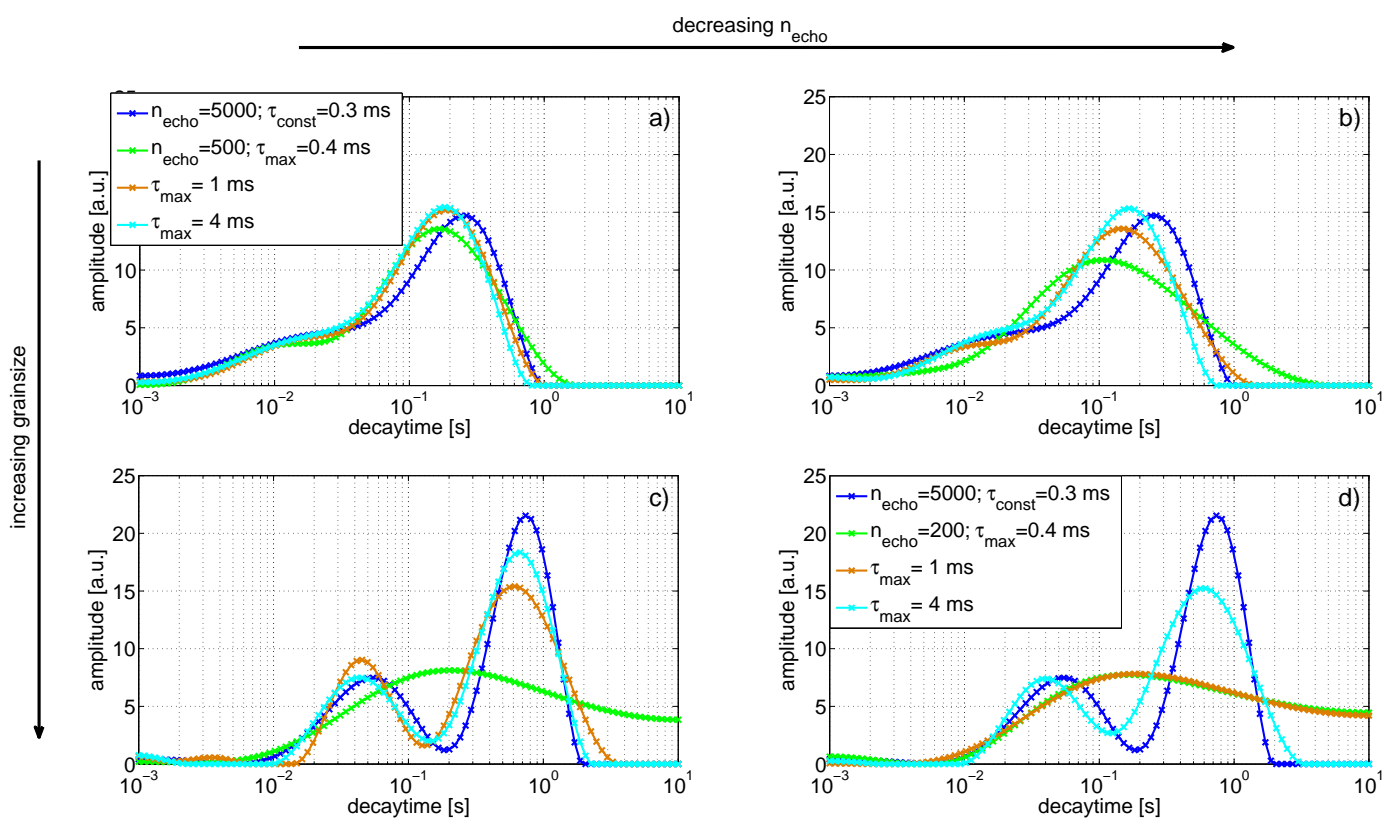

Fig. 8. $T_{2}$ distribution of granular material for pulse sequences with a linearly increased $\tau$. $n_{\text {echo }}$ is decreasing from left to right beginning $\left(n_{\text {echo }}=500-200\right)$. The grain size increases from top (medium sand) to bottom (coarse sand).

material, NMR signals decay more slowly due to a decrease of $\frac{S}{V}$ of the surface relaxation $T_{2, \mathrm{~S}}$. Thus, maintaining the sequence parameter $\left(n_{\text {echo }}, \tau_{\max }\right)$, the acquisition time becomes insufficiently short for slow decaying signals and small $\tau_{\max }$. Furthermore, the shorter acquisition time leads to a broadening of the distribution.

For decreasing $n_{\text {echo }}$ (from left to right) and fine-grained material, the same effect of broadening is observed; however, in addition to fully acquiring the signal, the sampling of the decay curve is the key factor. If the decay is completely acquired by the CPMG sequence, as it is the case for the fine-grained material and sequences with $\tau_{\max }=4000 \mu \mathrm{s}$, the reduction of data points due to decreased $n_{\text {echo leads to a }}$ broadening of the $T_{2}$ distributions. If the signal is not completely acquired, as it is the case for $\tau_{\max }=400 \mu \mathrm{s}$, a further broadening is observed.

For decreasing $n_{\text {echo }}$ and increasing the grain size, it shows that the effect of acquiring the complete signal dominates the data sampling effect. Theoretically a shortened acquisition time due to a reduction of $n_{\text {echo }}$ can be compensated by a larger $\tau_{\max }$; however, this leads to higher diffusion.

\subsubsection{Log mean decay time of glass beads}

The corresponding log mean decay times to the $T_{2}$ distributions of glass beads are given in Table 3. For each sample and amount of echos $\left(n_{\text {echo }}\right)$, a systematic decrease of $T_{2, \mathrm{lg}}$ for an increasing $\tau_{\max }$ is observable. On the one hand, this can be explained by an attenuated diffusion for fast decaying signals, which are fully acquired. On the other hand, for slower decaying signals the decrease of $T_{2, \mathrm{lg}}$ results from the broadening of the $T_{2}$ distribution due to an insufficient acquisition time.

If $n_{\text {echo }}$ is reduced $T_{2, \mathrm{lg}}$ decreases for silt and medium sand samples with $\tau_{\max }=1000 / 4000 \mu$ s, which is also an effect of acquisition time. In contrast, the sequence with $\tau_{\max }=400 \mu \mathrm{s}$ of medium sand and all sequences of coarse sand samples show an increasing $T_{2, \mathrm{lg}}$ if $n_{\text {echo }}$ decreases. This is a result of a flattened $T_{2}$ distribution with large decay times (beyond $3 \mathrm{~s})$ fitted to explain the data.

Sequences conducted for example with $n_{\text {echo }}=500$ and $\tau_{\max }=400 \mu \mathrm{s}$ seems to overestimate $T_{2, \mathrm{lg}}$ compared to the reference sequence (silt and medium-grained glass beads in Table 3), although the decay process is recorded completely. Compared to the reference sequence, fast decaying components vanish in the $T_{2}$ distributions (see Fig. 7). A cut-off time for calculating $T_{2, \mathrm{lg}}$ could possibly solve this problem.

\subsubsection{Comparison of $T_{2}$ distributions between samples of granular material and glass beads}

The $T_{2}$ distributions for samples of granular material are illustrated in Fig. 8. Sequences with $n_{\text {echo }}=50$ are neglected because the $T_{2}$ distribution of glass beads (see Fig. $7 \mathrm{c}, \mathrm{f}$ and i) showed that the acquisition time is too short for getting appropriate results. Also, the results for fine gravel are neglected because these large grained materials are assumed to have already good hydraulic properties, whose estimation was one of the major focuses.

In comparison to the glass beads, the $T_{2}$ distribution of granular material exhibits a bi-exponential behaviour that is more distinctive at coarse sand (Fig. 8c and d). Consistent 
with the glass beads, the shift of the $T_{2}$ distribution to shorter decay times is observed. In contrast to the fine material of glass beads (see Fig. 7a), the shift becomes more distinctive for decreasing $\tau_{\max }$. It can be assumed that an insufficient acquisition time caused this effect, since an increased diffusion term leads to an increasing shift for larger $\tau_{\max }$.

The absolute shift also increases with grain size, which could be an interaction of attenuated diffusion for increasing grain size and a short acquisition time. According to Fig. 8a and $\mathrm{c}$, a flattening of the $T_{2}$ distribution for coarse-grained material is observed.

A reduction of $n_{\text {echo }}$ shortens the record time and thus leads to broadened $T_{2}$ distributions. Additionally, as a result, the bi-exponential behaviour vanishes.

\subsubsection{Log mean decay time for samples of granular material}

In agreement with the results of glass beads, $T_{2, \text { lg }}$ of granular material (see Table 4) decreases if $\tau_{\max }$ increases and is explainable in the same way. Due to an insufficient acquisition time, $T_{2, \mathrm{gg}}$ increases for sequences with $\tau_{\max }=400$ and $1000 \mu$ s of coarse sand and CPMG sequence with $\tau_{\max }=400 \mu$ s of medium sand.

Apparently, $T_{2, \text { lg }}$ of the medium-grained granular material (first row in Table 4) is quite similar to the log mean decay time of fine-grained glass beads (Table 3 , first row). This similarity between these measurements could be explained by a difference in the surface relaxivity and/or internal magnetic field gradients. Moreover, even though the mean grain size is different, the $S / V$ may not be that different since glass beads have rather smooth surface and are spherical while the granulate material has a rough surface and grains are more angular.

\section{Conclusions}

As part of a project for developing an NMR borehole tool to characterize near surface groundwater layer in unconsolidated material, optimized CPMG sequences for measuring $T_{2}$ decay time were examined.

A synthetic study of sequences with an exponentially, logarithmically-equidistant and linearly-increased echo spacing showed that decay times of mono- and multiexponential signals can be resolved with respect to the sequence parameters. It can also be seen that the sequence with a linearly increasing $\tau$ yields the best results for slow and fast decaying components if diffusion is taken into account.

Laboratory measurements on glass beads and granular material of different grain size spectra showed that the main sequence parameters $\left(n_{\text {echo }}\right.$ and $\left.\tau_{\max }\right)$ cannot be chosen arbitrarily. Regarding the usage of NMR data, for example to estimate the hydraulic conductivity, a sequence with 500200 echos and $1000 \mu \mathrm{s} \leq \tau_{\max } \leq 2500 \mu \mathrm{s}$ is an appropriate
Table 4. Logarithmic mean decay time $T_{2, \mathrm{lg}}$ for all CPMG sequences conducted on samples granular material. The second column denotes $T_{2, \lg }$ of the reference sequence conducted with $n_{\text {echo }}=5000$ and a constant $\tau=300 \mu$ s.

\begin{tabular}{lrrrr}
\hline \multicolumn{1}{c}{ sample } & $\begin{array}{c}T_{2, \mathrm{lg}}[\mathrm{ms}] \\
\text { (reference) }\end{array}$ & $\tau_{\max }[\mu \mathrm{s}]$ & $\begin{array}{r}n_{\text {echo }}: 500 \\
\left(T_{2, \mathrm{lg}}[\mathrm{ms}]\right)\end{array}$ & $\begin{array}{c}200 \\
\left(T_{2, \mathrm{lg}}[\mathrm{ms}]\right)\end{array}$ \\
\hline $\mathrm{mS}$ & & 400 & 100.22 & 102.91 \\
$(250-500 \mu \mathrm{m})$ & 89.04 & 1000 & 93.9 & 88.46 \\
& & 4000 & 84.75 & 77.25 \\
\hline $\mathrm{gS}$ & & 400 & 326.84 & 343.51 \\
$(1000-1600 \mu \mathrm{m})$ & 264.15 & 1000 & 268.97 & 346.18 \\
& & 4000 & 262.47 & 264.3 \\
\hline
\end{tabular}

choice. Because coarse-grained material is of minor interest due to the high hydraulic conductivity, slow decaying signals may be estimated with higher uncertainty. Although, by adapting the sequence parameters it is possible to fit NMR data of coarse material with an acceptable accuracy. Therefore, an application of a CPMG sequence with a variable echo spacing to obtain slow and fast decaying components instead of using two CPMG sequences seems to be feasible, which results in faster measurements and thus an increased vertical resolution. A field test within an calibration pit is required to show the applicability under borehole conditions. Further investigation is needed to examine the behaviour on natural material. A closer look at the influence of different magnetic gradients on CPMG sequences with an increasing $\tau$ is of high interest.

Acknowledgements. We thank our project partners and colleagues for the discussions during the data acquisition and processing and for all contributions to improve this paper. The project was financed by the BMBF (Federal Ministry of Education and Research).

Edited by: L. Vazquez

\section{References}

Aster, R., Borchers, B., and Thurber, C.: Parameter Estimation and Inverse Problems, Elsevier Academic Press, 2005.

Bloch, F., Hansen, W. W., and Packard, M.: The Nuclear Induction Experiment, Phys. Rev., 70, 474-485, doi:10.1103/PhysRev.70.474, 1946.

Brown, R. and Gamson, B.: Nuclear, magnetism logging, J. Petrol. Technol., 12, 199-207, 1960.

Brownstein, K. R. and Tarr, C. E.: Importance of classical diffusion in NMR studies of water in biological cells, Phys. Rev. A, 19, 2446-2453, doi:10.1103/PhysRevA.19.2446, 1979.

Carr, H. Y. and Purcell, E. M.: Effects of Diffusion on Free Precession in Nuclear Magnetic Resonance Experiments, Phys. Rev., 94, 630-638, doi:10.1103/PhysRev.94.630, 1954.

Coates, G., Xiao, L., and Prammer, M.: NMR Logging Principles and Application, Halliburton Energy Services, Houston, 1999. 
Dunn, K. J., Bergman, D. J., and Latorraca, G. A.: Nuclear magnetic resonance, 1st Edn., Pergamon, 2002.

Godefroy, S., Fleury, M., Deflandre, F., and Korb, J.-P.: Temperature effect on NMR surface relaxation, in: SPE Annual Technical Conference and Exhibition, 30 September-3 October 2001, New Orleans, Louisiana, doi:10.2118/71700-MS, 2001.

Hahn, E. L.: Spin Echoes, Phys. Rev., 80, 580-594, doi:10.1103/PhysRev.80.580, 1950.

Hürlimann, M. D. and Venkataramanan, L.: Quantitative Measurement of Two-Dimensional Distribution Functions of Diffusion and Relaxation in Frossly Inhomogeneous Fields, J. Magnet. Reson., 157, 31-42, doi:10.1006/jmre.2002.2567, 2002.

Kenyon, W. E.: Petrophysical Principles of Applications of NMR Logging, Log Analyst, 38, 21-43, 1997.

Kleinberg, R. L.: Pore size distributions, pore coupling, and transverse relaxation spectra of porous rocks, Proceedings of the Second International Meeting on Recent Advances in MR Applications to Porous Media, Magnet. Reson. Imaging, 12, 271-274, doi:10.1016/0730-725X(94)91534-2, 1994.

Kleinberg, R. L. and Horsfield, M. A.: Transverse relaxation processes in porous sedimentary rock, J. Magnet. Reson., 88, 9-19, doi:10.1016/0022-2364(90)90104-H, 1990.

Kruspe, T., Thern, H. F., Kurz, G., Blanz, M., Akkurt, R., Ruwaili, S., Seifert, D., and Marsala, A. F.: Slimhole Application of Magnetic Resonance While Drilling, in: SPWLA 50th Annual Logging Symposium, 21-24 June 2009, The Woodlands, Texas, p. $15,2009$.
Meiboom, S. and Gill, D.: Modified Spin-Echo Method for Measuring Nuclear Relaxation Times, Rev. Sci. Instrum., 29, 688-691, doi:10.1063/1.1716296, 1958.

Miller, M., Paltiel, Z., Gillen, M., Granot, J., and Bouton, J.: Spin Echo Magnetic Resonance Logging: Porosity and Free Fluid Index Determination, SPE paper 20561, New Orleans, Louisiana, 1990.

Mohnke, O. and Yaramanci, U.: Pore size distributions and hydraulic conductivities of rocks derived from Magnetic Resonance Sounding relaxation data using multi-exponential decay time inversion, J. Appl. Geophys., 66, 73-81, doi:10.1016/j.jappgeo.2008.05.002, 2008.

Purcell, E. M., Torrey, H. C., and Pound, R. V.: Resonance Absorption by Nuclear Magnetic Moments in a Solid, Phys. Rev., 69, 37-38, doi:10.1103/PhysRev.69.37, 1946.

Seevers, D.: A nuclear magnetic method for determining the permeability of sandstones, Paper L, Society of Professional Well Log Analysts Transactions, Tulsa, Oklahoma, 1-14, 1966.

Song, Y.-Q.: Using internal magnetic fields to obtain pore size distributions of porous media, Concept. Magnet. Reson. A, 18, 97 110, doi:10.1002/cmr.a.10072, 2003.

Torrey, H. C.: Bloch Equations with Diffusion Terms, Phys. Rev., 104, 563-565, doi:10.1103/PhysRev.104.563, 1956.

Vista Clara, I.: Javelin - Manual and Documentation, 1.2 Edn., Vista Clara, Inc., Mukilteo, USA, 2011. 